\title{
PERFORMANCE EVALUATION OF AN IMPROVED PRMA PROTOCOL FOR LOW EARTH ORBIT MOBILE COMMUNICATION SYSTEMS
}

\author{
enrico del re*, romano fantacci, giovanni giambene and sergio walter \\ Dipartimento di Ingegneria Elettronica, Universita degli Studi di Firenze, Via S. Marta 3, I-50139 Firenze, Italy
}

\begin{abstract}
SUMMARY
Future mobile communication systems will be characterized by the interworking of several networks that will be integrated into a unique system. The satellite component and the terrestrial one will use as far as possible the same protocols. This work is concerned with Medium Access Control (MAC) protocols. In particular, Packet Reservation Multiple Access (PRMA) has been considered as a good candidate for terrestrial cellular systems, since it allows high multiplexing gains, dynamic PRMA carrier allocation to cells, easy management of integrated voice and data traffic and a near-transparent behaviour with respect to user mobility. The main aim of this preliminary work is to investigate the suitability of the PRMA protocol for application to Low Earth Orbit Mobile Satellite Systems (LEO-MSSs). Moreover, we have proposed a novel version of the PRMA protocol, named PRMA with Hindering States (PRMA-HS), that is particularly suitable for application in LEO systems. This new technique has shown very promising results in terms of both packet dropping probability and throughput. (C) 1997 John Wiley \& Sons, Ltd.
\end{abstract}

key words: mobile satellite systems; LEO satellites; PRMA

\section{INTRODUCTION}

Mobile communications is the fastest growing area within the telecommunications sector. By the year 2010 about 40 per cent of the European population will have personal communications for private use. ${ }^{1}$ A similar behaviour is expected all over the world.

Future third-generation mobile communication systems are in the specification phase within the European Telecommunications Standards Institute (ETSI) under the name Universal Mobile Telecommunications Systems (UMTS). The equivalent denomination of the International Telecommunications Union (ITU) is International Mobile Telecommunications after the year 2000 (IMT-2000). Basic characteristics of UMTS are ${ }^{2}$ terminal and personal mobility, global coverage, global roaming, multimedia and personalized services with applications up to $2 \mathrm{MBit} / \mathrm{s}$. The two worldwide radio frequency bands allocated to UMTS are 1885-2025 and $2110-2200 \mathrm{MHz}$.

The scenario envisaged in this work is the future UMTS, where a terrestrial component will be integrated with a satellite one at the system level: both

\footnotetext{
*Correspondence to: E. Del Re, Dipartimento di Ingegneria Elettronica, Universita degli Studi di Firenze, Via S. Marta 3, I50139 Firenze, Italy. Email: delre@lenst.die.unifi.it Contract grant sponsor: Italian Space Agency Contract grant sponsor: MURST
}

networks will use as far as possible the same protocols at the different layers of the OSI stack. ${ }^{3}$ The purpose of this paper is to investigate the suitability of the same multiple access scheme for application in both terrestrial and satellite networks ${ }^{4}$ in order to simplify the integration of the two systems.

The Packet Reservation Multiple Access (PRMA) protocol $^{5,6}$ has been proposed for terrestrial microcellular networks, where it has exhibited very interesting features in the light of the future UMTS: management of voice and data traffic for multimedia applications, ${ }^{7,8}$ compatibility with the ATM standard, ${ }^{9}$ high efficiency expressed by a high multiplexing gain value, dynamic PRMA carrier allocation to cells, ${ }^{10,11}$ support of multirate applications and a more transparent behaviour with respect to user mobility. Recently, many variants have been proposed for the PRMA protocol, which prove an increasing interest in such multiple access techniques. ${ }^{12-15}$

The satellite segment of the future UMTS will be based on non-GEO satellites; in particular, we assume here a Low Earth Orbit Mobile Satellite System (LEO-MSS), since it is characterized by low propagation delays and low propagation attenuations that allow the use of low-power hand-held mobile terminals. $^{4}$ Figure 1 presents the relationship between the maximum value of the Round Trip Delay, $R T D_{\max }$, experienced by a User Terminal (UT) for a satellite altitude $A_{\mathrm{s}}$ and a minimum elevation angle $E l_{\min }$. 


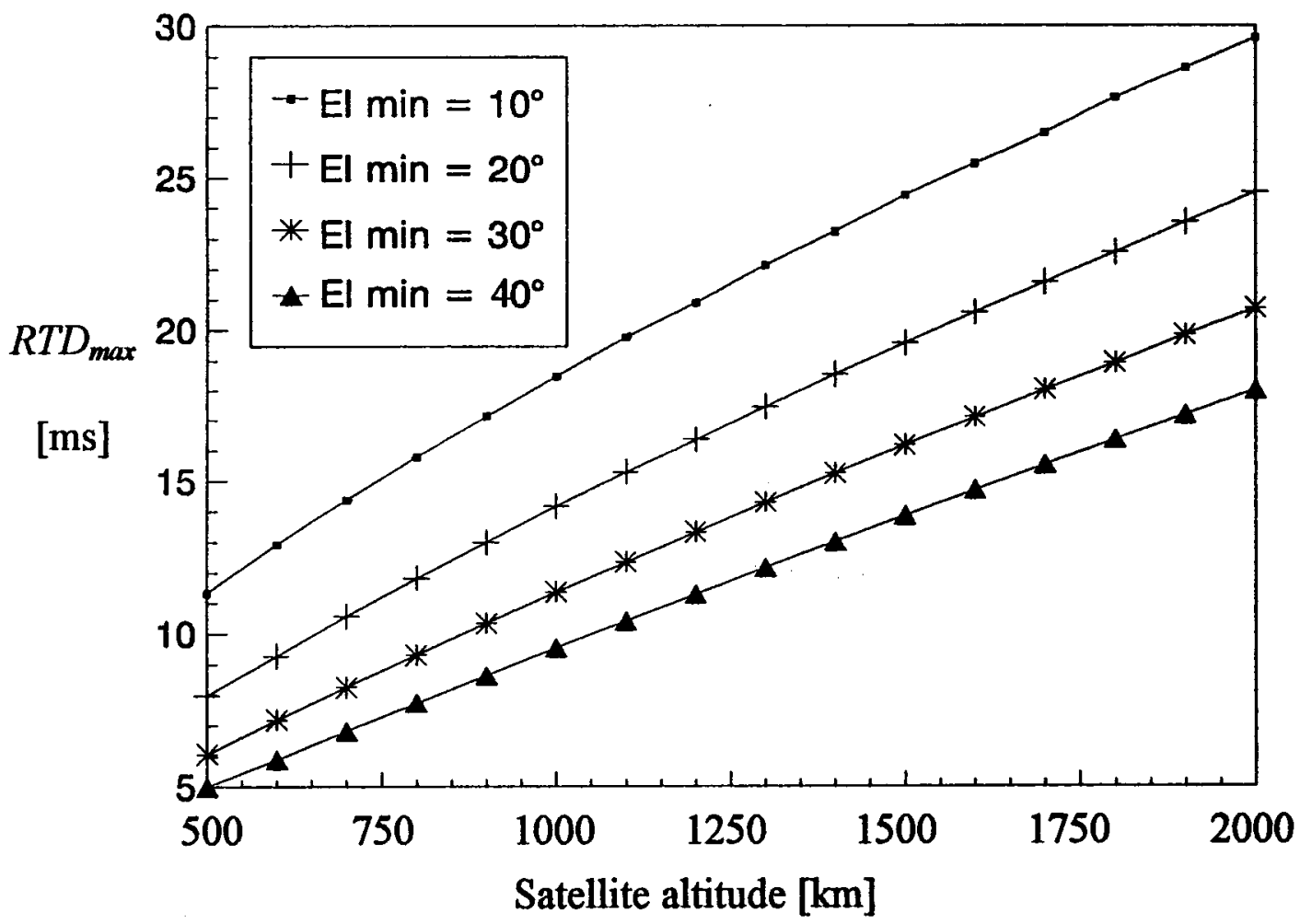

Figure 1. Behaviour of $R T D_{\max }$ as a function of LEO satellite altitude for various values of minimum elevation angle

This work is a preliminary study which investigates the use of the PRMA protocol for voice traffic in order to efficiently manage resources on the uplink in LEO-MSSs (i.e. from UTs to satellite). Of course, a suitable multiplexing technique must also be used on the downlink (e.g. a Digital Speech Interpolation technique $\left.{ }^{16}\right)$. Moreover, this work proposes an evolution of the PRMA protocol, termed PRMA with Hindering States (PRMA-HS), particularly suitable for both the terrestrial and the satellite segment of UMTS: we will prove that this protocol maintains the same behaviour as the classical PRMA scheme in terrestrial cellular systems, whereas it attains a significant capacity increase in the LEO-MSS environment.

In LEO-MSSs two solutions have been proposed in order to define the cells on the earth: one is called satellite-fixed cells, where cells are fixed with respect to the satellite and move as regards the earth; the other is called earth-fixed cells, where cells are fixed on the earth (satellite antenna spotbeams are steered so as to point to the same cells on the earth as long as possible; this is the solution of the TELEDESIC system ${ }^{17}$ ).

The first solution has been adopted, for instance, by the IRIDIUM system, ${ }^{18}$ where the satellite ground-track speed is about $24,000 \mathrm{~km} \mathrm{~h}^{-1}$ and the average spot-beam footprint (=cell) radius is about $300 \mathrm{~km}$. As a result, cells move fast with respect to the earth. ${ }^{19}$ It has been estimated that, on average, a hand-off between spots of the same satellite occurs about every $1 \mathrm{~min}$ and a hand-off between spots belonging to different satellites occurs about every 6 min.
In the second case the earth has been mapped into a fixed grid of cells. There is no impact of the satellite motion (or it can be neglected) on cell changes for the users. Therefore the user mobility only depends on the users' speed. Assuming a cell diameter of $54 \mathrm{~km}$ as in the TELEDESIC system, the hand-off occurrence may be neglected at typical vehicular speeds (maximum speed equal to $\left.100 \mathrm{~km} \mathrm{~h}^{-1}\right)$.

In this work we assume an earth-fixed cell system, so we do not consider aspects related to mobility. However, even if the satellite-fixed cell approach were assumed, the hand-off management with PRMA should not pose significant problems: a UT sending a hand-off request is considered as a UT starting a talkspurt in the destination cell.

This paper is organized as follows. Section 2 presents an overview of the classical PRMA protocol in terrestrial cellular systems. Section 3 proposes the application of the original PRMA protocol to MSSs. Then a novel version of the PRMA protocol (i.e. PRMA-HS) particularly suitable for LEO-MSSs is described in Section 4 and its performance is obtained by simulations in Section 5 .

\section{OVERVIEW OF THE CLASSICAL PRMA PROTOCOL}

A PRMA carrier $^{5,6}$ is divided into time intervals, called slots, with duration $T_{\mathrm{s}}$; they are grouped in blocks of $N$ slots and each group is a frame of duration $T_{\mathrm{f}}$. Homologous slots in subsequent frames form a communication channel. The access to a channel is random, similarly to slotted ALOHA $;{ }^{20}$ 
once a UT obtains access to a channel, it has reservation for the exclusive use of a slot per frame as in the TDMA scheme.

The transmission of voice is organized in packets; each packet is composed of user information bits and a header which contains routing, synchronization and control information. The PRMA protocol is based on a speech activity detector which distinguishes silent gaps within a conversation. As soon as a silent gap has been revealed, the UT immediately releases the reservation of its slot. Therefore another UT which needs to transmit a talkspurt can utilize this slot. Hence a larger number of active UTs than the physical number of channels (i.e. the number of slots per frame) can be simultaneously served. At the beginning of each talkspurt the related UT has to contend against other UTs in order to obtain a reservation according to a random access scheme: the UT enters the contending state. In this work we have assumed a slow speech activity detector ${ }^{21}$ which reveals only principal gaps within the conversation. We consider that a fast speech activity detector does not attain a good performance in a satellite environment since it entails a greater contention rate, which has to be avoided in the presence of high RTD values.

A feedback channel broadcast by the controller (in the terrestrial case, the base station; in the satellite case, the satellite itself which has onboard processing capability) informs all the UTs within a cell about the state of each slot of the PRMA carrier (i.e. idle/reserved) and, for a reserved slot, the UT which is currently using it.

If two or more UTs try to send their first packets on the same slot, there is a collision. If we neglect the capture effect, ${ }^{20,21}$ the controller cannot recognize any user, so it leaves the slot unreserved. If all the UTs were to try again on the next free slot, there would be another packet collision, and so on. To avoid this dangerous loop, a UT which has a talkspurt to transmit is enabled to transmit its packet on a free slot according to a permission probability p. Therefore, after a collision has occurred, the involved UTs reschedule their transmission attempts on free slots in a random way.

A UT in the contending state discards the first packet when the access delay (i.e. the time to obtain a reservation) exceeds a maximum value $D_{\max }$. Experimental tests have shown that an acceptable value of $D_{\max }$ is $32 \mathrm{~ms}$ for packets that contain up to $10-20 \mathrm{~ms}$ of voice. ${ }^{22}$ If the first packet of a talkspurt does not obtain a reservation within $D_{\max }$, it is discarded; then the associated UT tries to transmit the second packet of its talkspurt and so on.

The quality of the voice transmission with PRMA is measured by the probability $P_{\text {drop }}$ that a packet is discarded by the buffer of a UT because it has not obtained a reservation within $D_{\max }$. Obviously, the greater the number of UTs with a call in progress that share a PRMA carrier, $M$, the greater is the average number of UTs in the contending state and the greater is $P_{\text {drop. }}$. The requirement $P_{\text {drop }} \leq 1 \%$ must be fulfilled to have a minimal degradation in perceivable speech quality in contemporary speech codecs. $^{21,22}$ Therefore the capacity of a PRMA carrier, $M_{0.01}$, is defined as the maximum number of users with a call in progress that may share a PRMA carrier with $P_{\text {drop }} \leq 1 \%$.

A further performance parameter is represented by the throughput $\eta$, which is the average number of packets that are successfully transmitted per slot. The ideal maximum value of $\eta$ is 1 packet/slot, but this is only possible in the case of deterministic traffic. In terrestrial cellular systems, through a suitable selection of the parameter values, the PRMA protocol attains high values of $\eta$, close to 0.75 packet/slot, in correspondence with the maximum capacity $M_{0 \cdot 01} \cdot{ }^{21}$

The frame structure is designed in such a way that a speech source generates exactly one packet per frame. According to this, it follows that

$$
N=\left\lfloor\frac{R_{\mathrm{c}} T_{\mathrm{f}}}{R_{\mathrm{s}} T_{\mathrm{f}}+H_{\mathrm{v}}}\right\rfloor \text { slots/frame }
$$

where $R_{\mathrm{c}}, R_{\mathrm{s}}$ and $H_{\mathrm{v}}$ are as defined in Table I and $\lfloor x\rfloor$ denotes the highest integer number less than or equal to $x$. Note that Table I also presents the system parameter values assumed in this work. These values have changed with respect to Reference 21 for reasons that will be clarified in the next section; in particular, the value of $R_{\mathrm{s}}$ is not varied (it is related to the G.721 voice codec), whereas $R_{\mathrm{c}}$ has increased slightly.

Practical values of $R_{\mathrm{s}}$ in satellite systems can be lower than that considered in Table I. However, we will retain such a value for this study which aims to prove the suitability of PRMA protocols for application in LEO-MSSs.

The slot duration $T_{\mathrm{s}}$ and the maximum access delay for a voice packet expressed in slots, $D$, can be derived as follows, once $T_{\mathrm{f}}$ has been selected and $N$ has been obtained from (1):

$$
T_{\mathrm{s}}=\frac{T_{\mathrm{f}}}{N}, \quad D=\left\lceil\frac{D_{\max }}{T_{\mathrm{s}}}\right\rceil \text { slots }
$$

where $\lceil x\rceil$ denotes the smallest integer number greater than or equal to $x$. For instance, according to (1) and on the basis of the values in Table I, we

Table I. Parameter definitions and values used in this work

\begin{tabular}{lll}
\hline Parameter & \multicolumn{1}{c}{ Definition } & Value \\
\hline$R_{\mathrm{c}}$ & Channel bit rate & $765 \mathrm{kbit} / \mathrm{s}$ \\
$R_{\mathrm{s}}$ & Speech source bit rate & $32 \mathrm{kbit} / \mathrm{s}$ \\
$H_{\mathrm{v}}$ & Header size of a packet & $64 \mathrm{bit}$ \\
$D_{\max }$ & Maximum access delay & $32 \mathrm{~ms}$ \\
& for a voice packet & \\
\hline
\end{tabular}


obtain $N=21$ slots $/$ frame for $T_{\mathrm{f}}=15 \mathrm{~ms}$. Consequently, from (2) we have $T_{\mathrm{s}}=0.71 \mathrm{~ms}$ and $D=46$ slots.

Figure 2 presents the terminal state diagram proposed in Reference 21 to model the UTs' behaviour. In this figure the following states have been shown: the SIL state when a UT is in a silent phase; the $\operatorname{RES}_{i}$ states $(i=0, \ldots, N-1)$ where a UT has a reservation; the contending state $\mathrm{CON}$ for a UT. We have also considered a transition from the CON state to the SIL one with rate $\gamma$ that has been neglected in the model shown in Reference 21 for the slow speech activity detector. This transition takes into account that a talkspurt may end before obtaining a reservation.

The symbols used in Figure 2 are defined as follows: $\sigma$ is the probability that a silent gap with mean duration $t_{2}$ ends within $T_{\mathrm{s}} ; \gamma$ is the probability that a talkspurt with mean duration $t_{1}$ ends during $T_{\mathrm{s}} ; \gamma_{\mathrm{f}}$ is the probability that a talkspurt ends within $T_{\mathrm{f}} ; a$ is the probability that a UT attempts to transmit on the next slot; $u$ is the probability that a transmission attempt is successful. More details are given in Reference 21.

According to the voice source model described in Reference 21, talkspurt and silent periods are exponentially distributed with mean values $t_{1}$ and $t_{2}$ respectively. Typical values are $1 \mathrm{~s}$ for $t_{1}$ and $1.35 \mathrm{~s}$ for $t_{2 \cdot}{ }^{21}$

Let us introduce the multiplexing gain parameter $\mu_{0.01}$, which is the ratio between the PRMA carrier capacity $M_{0.01}$ and the equivalent capacity of a TDMA carrier (ideal case without overload), $R_{\mathrm{c}} / R_{\mathrm{s}}$ :

$$
\mu_{0 \cdot 01}=\frac{M_{0 \cdot 01} R_{\mathrm{s}}}{R_{\mathrm{c}}} \text { conversations } / \text { channel }
$$

Only when $\mu_{0.01}$ is greater than unity does the PRMA protocol outperform the TDMA scheme. The upper bound for $\mu_{0.01}$ is given by $\left(t_{2}+t_{1}\right) / t_{1} \approx 2 \cdot 25$ conversations/channel. This parameter clearly expresses the efficiency of the PRMA technique. In terrestrial cellular systems, with optimized parameter values, $\mu_{0.01}$ may reach 1.7 conversations/channel. ${ }^{21}$

Research efforts have been carried out in order to theoretically predict the behaviour of the PRMA protocol in terms of several performance parameters such as $P_{\text {drop }}$ and the throughput $\eta .^{21,23,24}$

\section{APPLICATION OF THE PRMA PROTOCOL TO A SATELLITE COMMUNICATION SYSTEM: A FEASIBILITY STUDY}

In order to apply the PRMA protocol to an MSS, we must take account of the RTD, i.e. the time needed to know the outcome of a packet transmission attempt during the contention phase; this time is much greater than that experienced in terrestrial cellular systems (e.g. $6 \mu$ s for a terrestrial cell with $1 \mathrm{~km}$ radius) and is not negligible with respect to the slot duration.

Some preliminary considerations about the application of the PRMA protocol in MSSs appeared in Reference 25. The high RTD value in the case of geostationary satellites prevents any application of the PRMA in such a case (e.g. the RTD is equal to $250 \mathrm{~ms}$ for a user which sees the satellite at the zenith). ${ }^{25}$ Therefore we will limit our study to the case of non-geostationary MSSs that are at lower altitudes (and, then, with lower RTD values) than geostationary satellites. In particular, this work is focused on LEO satellites that may guarantee very low propagation delays in the range $5-30 \mathrm{~ms}$ (see Figure 1). For instance, let us consider a LEO satellite constellation with $R T D_{\max }=10 \mathrm{~ms}$; this means that a contending UT has up to three attempts to transmit the first packet of its talkspurt before dropping it. In this section we will also prove that the higher RTD values of Medium Earth Orbit (MEO) constellations prevent the PRMA application.

The easiest way to extend the PRMA protocol to LEO-MSSs is to consider that when a UT makes a

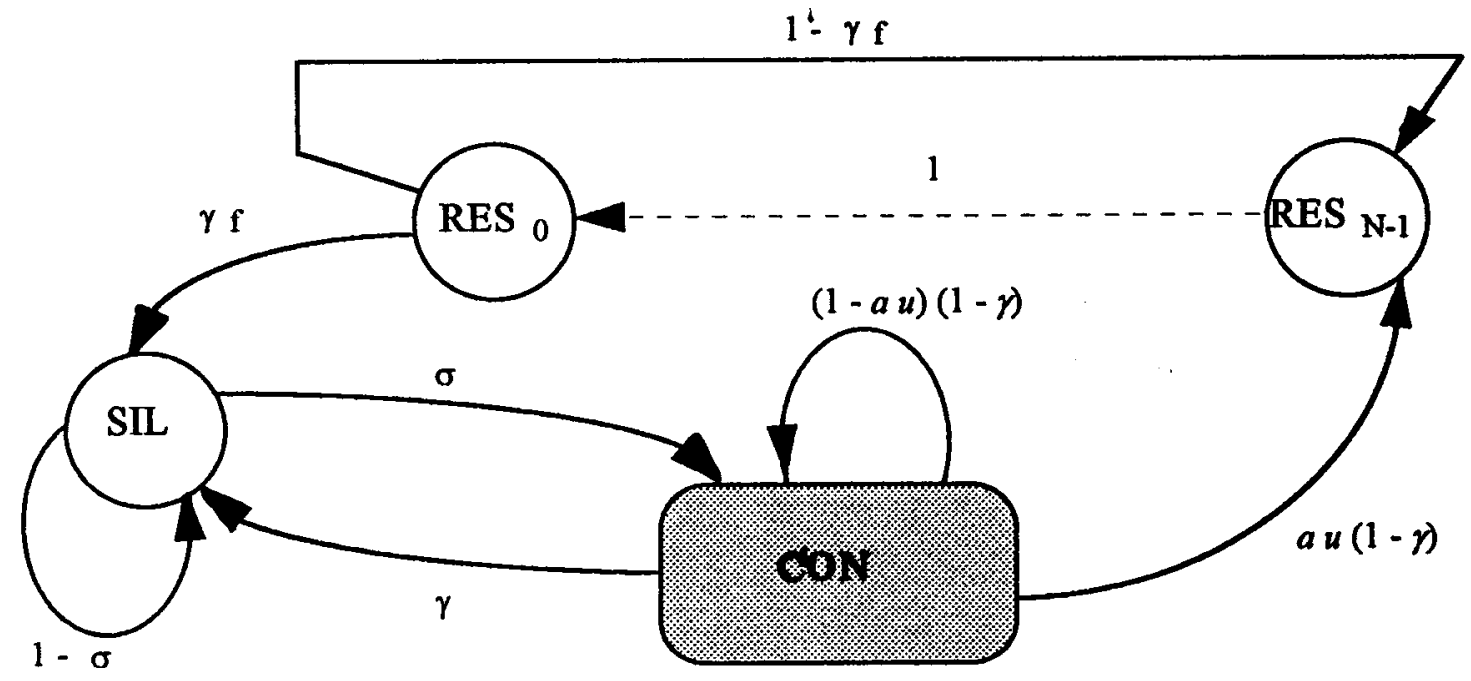

Figure 2. Terminal state diagram for terrestrial PRMA protocol 
transmission attempt, it leaves the contending state CON (i.e. it stops contending) and waits for the result of its attempt sent by the satellite and received after an RTD time. Throughout this paper we have considered that the RTD is always equal to its maximum value $R T D_{\max }$ (conservative assumption).

In the preliminary investigation carried out in this section, we have selected $T_{\mathrm{f}}$ equal to $R T D_{\max }$ for the sake of simplicity (in the next sections we will remove such a constraint); therefore, when a UT makes an acquisition attempt, it leaves the contending state and enters a block of waiting states $\left(\mathrm{WAIT}_{i}\right)$ that model the delay to receive the information about the outcome of its transmission attempt. The UT leaves the waiting states when the RTD has ended. At this time the UT knows the result of its attempt; then it enters the reservation states $\mathrm{RES}_{i}$ or it goes back to the contending state $\mathrm{CON}$, depending on whether the transmission attempt has been successful or not. In this work we do not consider the effect of packet transmission errors due to the channel.

We need to study the impact of different values of both $T_{\mathrm{f}}$ and $p$ on the PRMA performance. We assume the parameter values shown in Table I. Since satellites are both bandwidth- and power-limited, their spectrum resource has to be carefully exploited. As a consequence, practical implementations of the PRMA protocol via satellite should consider lower $R_{\mathrm{S}}$ values (e.g. four to eight times) than that shown in Table I for voice sources (i.e. $R_{\mathrm{s}}=32 \mathrm{kbit} / \mathrm{s}$, G.721 codec). However, owing to the statistical multiplexing characteristics of the PRMA protocol, it is expected that $\mu_{0.01}$ will increase if the number of equivalent TDMA channels increases (i.e. $\left.R_{\mathrm{c}} / R_{\mathrm{s}}\right) .{ }^{21}$ Therefore, if powerful voice codecs (i.e. with low $R_{\mathrm{s}}$ ) are used, greater advantages in terms of both capacity and multiplexing gain are possible with PRMA.

\subsection{Selection of $p$}

We have assumed $M=30$ users/carrier and considered both $T_{\mathrm{f}}=5$ and $15 \mathrm{~ms}$ (note that the first case has $T_{\mathrm{f}}=5 \mathrm{~ms}$, which, according to the assumption $T_{\mathrm{f}}=R T D_{\max }$, entails a limit situation for the altitude of LEO systems*). On the basis of the system parameter values shown in Table I, from (1) we have $N=17$ and 21 respectively. In Figure 3 we have shown the behaviour of $P_{\text {drop }}$ as a function of $p$ for both the terrestrial and the LEO satellite cases. The $P_{\text {drop }}$ values obtained with $T_{\mathrm{f}}=15 \mathrm{~ms}$ are far below those obtained with $T_{\mathrm{f}}=5 \mathrm{~ms}$ for both terrestrial and satellite systems. This is an interesting result which highlights that $T_{\mathrm{f}}=15 \mathrm{~ms}$ is a good

\footnotetext{
* The limit situation for LEO-MSSs with $R T D_{\max }=5 \mathrm{~ms}$ has been considered only with the goal to evaluate the PRMA performance in a LEO-MSS which presents the most favourable conditions for the RTD, very close to a terrestrial scenario.
}

choice for the PRMA protocol, as will be further discussed in the next subsection.

In the satellite case, regardless of the value of $T_{\mathrm{f}}$, we have that if $p$ increases from $0 \cdot 1$ to $0 \cdot 4$, the packet dropping probability decreases: the contention mechanism works better. A further increase in $p$ causes an increase in $P_{\text {drop }}$ owing to the high number of collisions involved in the contention phase. When $p$ is close to unity, the PRMA protocol does not work correctly, because two contending UTs will always collide on the next available slots, hence blocking the reservation mechanism. Moreover, it is evident that in both the satellite cases under examination (i.e. $T_{\mathrm{f}}=15$ and $5 \mathrm{~ms}$ ) the behaviours of $P_{\text {drop }}$ have a minimum around $p=0 \cdot 4$. Therefore this value has to be considered as a good choice over a wide range of $T_{\mathrm{f}}$ values.

For the terrestrial case we have also obtained that $P=0.4$ represents an optimum choice for the system parameter values assumed in Table I, regardless of the selected value of $T_{\mathrm{f}}$. As expected, the terrestrial case exhibits lower $P_{\text {drop }}$ values with respect to the satellite one. However, if $p$ approaches $0 \cdot 6$, probability $P_{\text {drop }}$ suddenly increases, because the contending state is congested (if $p$ is high, we have more collisions). This behaviour is different from that shown for the satellite PRMA, where the RTD prevents frequent contention attempts. However, probability $P_{\text {drop }}$ reaches unity when $p$ approaches zero or unity also in the satellite case.

In the satellite case, in the region of $p$ where $P_{\text {drop }}$ has a minimum for $M=30$ users/carrier, $P_{\text {drop }} \leq 1 \%$. If we let $M_{0.01}=30$ users/carrier in (3), we obtain a low multiplexing gain value: $\mu_{0.01} \approx 1.25$ conversations/channel. The following subsection presents further results which show the impact of the frame duration on the multiplexing gain performance of the PRMA protocol. This study may help the system designer in selecting a suitable frame duration.*

\subsection{Selection of $\mathrm{T}_{f}$}

An interesting topic is represented by the study of system performance as a function of the frame duration $T_{\mathrm{f}}$, assumed equal to $R T D_{\text {max }}$ in this section. Figure 4 shows the multiplexing gain $\mu_{0.01}$ as a function of $T_{\mathrm{f}}$ with $p=0.4$ for both the terrestrial and the satellite scenario. The system parameter values in Table I are kept fixed; then, according to (1), an increase in $T_{\mathrm{f}}$ causes longer packets. The impact of the variation of $T_{\mathrm{f}}$ on the performance of the PRMA protocol in LEO-MSSs can be explained as follows.

* Of course, also the selection of the voice codec has an impact on the frame duration value. The investigation of this aspect is beyond the scope of this paper, which aims to highlight the potentialities of the PRMA protocol in LEO-MSSs; therefore we consider a $32 \mathrm{kbit} / \mathrm{s}$ voice codec. 


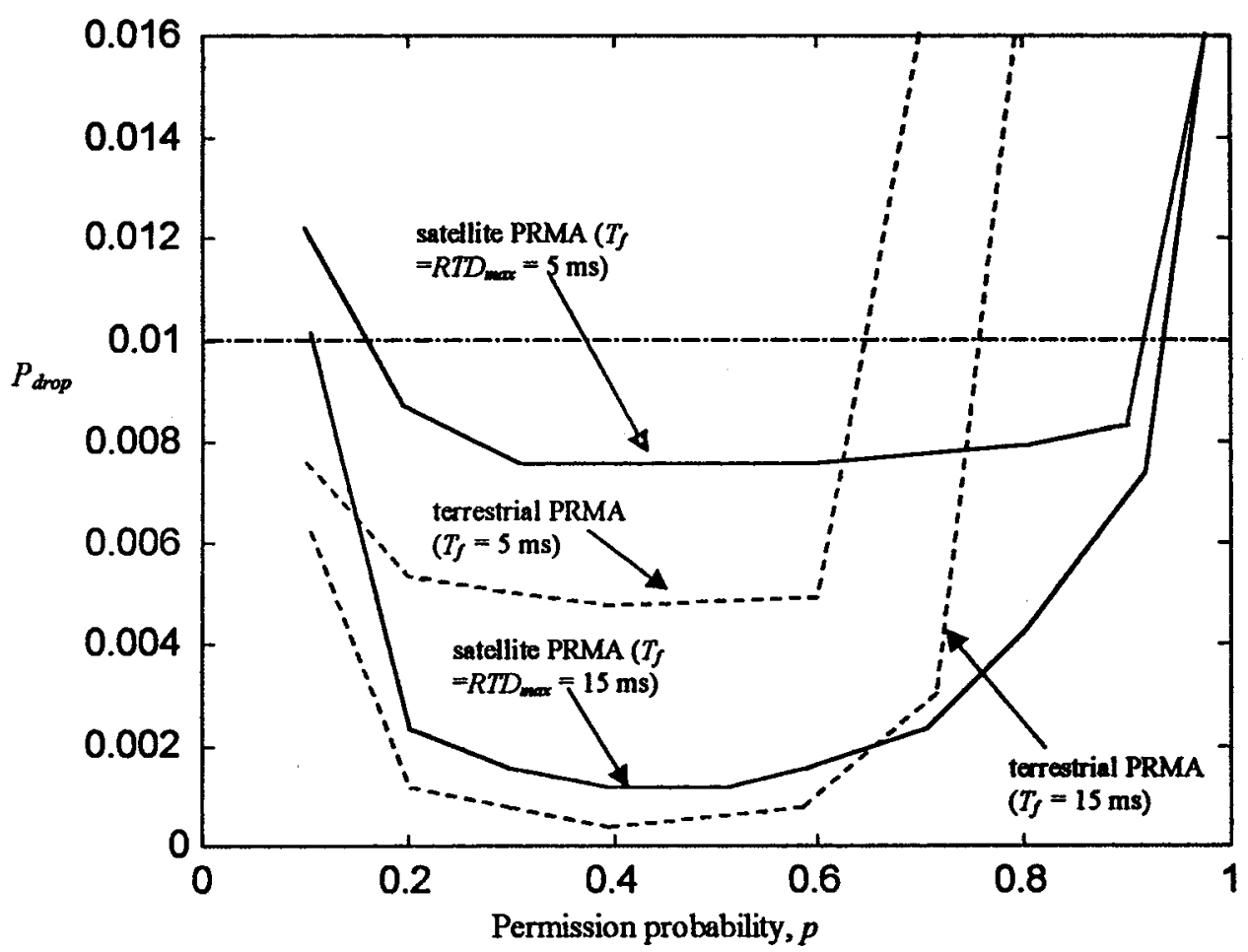

Figure 3. Behaviour of $P_{\text {drop }}$ versus $p$ with $M=30$ users/carrier for PRMA protocol in both LEO satellite and terrestrial cases

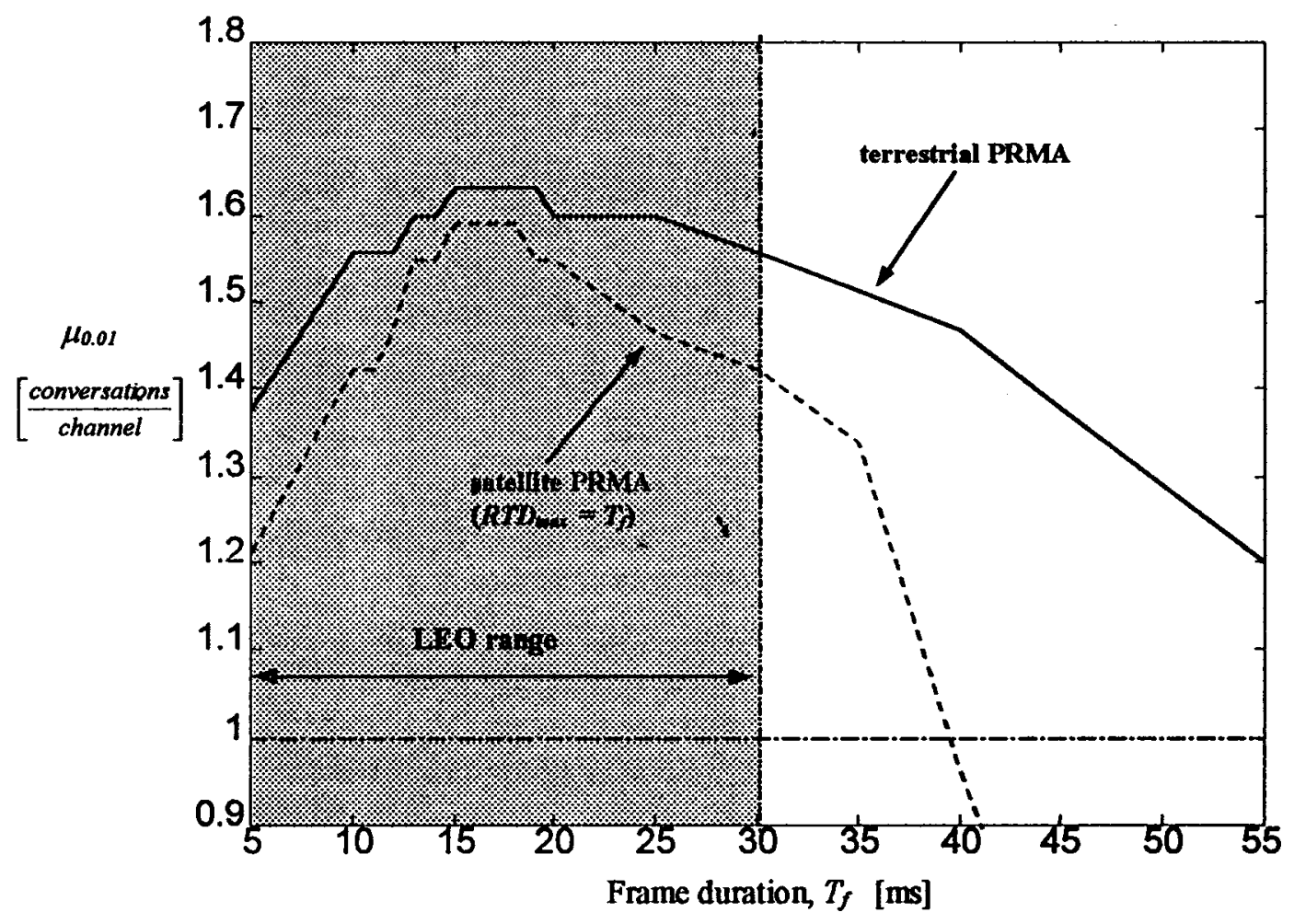

Figure 4. PRMA capacity as a function of $T_{\mathrm{f}}$ with $p=0 \cdot 4$ for both terrestrial and satellite cases

- A low value of $T_{\mathrm{f}}$ implies to reduce the slot duration, so a UT needs a large number of reserved slots to transmit its talkspurt. Then a UT in the transmitting state holds a reservation for many frame intervals, so reducing the number of slots available for the contentions of other users. In this way the multiplexing characteristics of the PRMA protocol are significantly reduced. Consequently $P_{\text {drop }}$ increases and then the number of simultaneous conversations supported per carrier decreases.

- An excessive value of $T_{\mathrm{f}}$ causes a worse system performance owing to the maximum acceptable delay $D_{\max }$ : a reduced number of attempts are 
available for the contending terminals in order to obtain a reservation, so many packets experience an access delay greater than $D_{\max }$ without obtaining a reservation. If a frame duration greater than $35 \mathrm{~ms}$ is considered, the multiplexing gain $\mu_{0.01}$ suddenly reduces and falls below unity; in this case the PRMA protocol loses its advantages with respect to TDMA.

From Figure 4 we note a significant difference between the terrestrial case and the satellite one for high values of $T_{\mathrm{f}}$ : in the satellite case we have a more evident reduction of $\mu_{0.01}$ when $T_{\mathrm{f}}$ exceeds $35 \mathrm{~ms}$. This difference is due to the high RTD value in the satellite case, which significantly reduces the number of possible contention attempts within $D_{\max }$. However, in both the terrestrial case and the satellite one, $\mu_{0.01}$ has a quite flat maximum for $T_{\mathrm{f}}=15-$ 16 ms.*

In LEO-MSSs the altitude varies from 500 to $2000 \mathrm{~km}$ and, according to Figure 1, the $R T D_{\max }$ values vary from 5 to $30 \mathrm{~ms}$ (depending on the minimum elevation angle), whereas MEO-MSSs are characterized by an altitude in the range 10,000$13,000 \mathrm{~km}$ and $R T D_{\max }$ values greater than $70 \mathrm{~ms}$. If we consider these $R T D_{\max }$ values and the behaviour shown in Figure 4, we may conclude that the PRMA protocol is suitable for application in LEO systems (i.e. $\mu_{0.01}>1$ ) but not in MEO ones.

In conclusion, it is possible to select the same optimum value of $p=0.4$ and the same optimum frame duration of $15 \mathrm{~ms}$ in both terrestrial and satellite systems. This is a promising result in the light of the future expected integration between terrestrial and satellite systems.

\section{THE PROPOSED PRMA-HS PROTOCOL}

With PRMA, after a transmission attempt a UT stops contending to wait for the result received from the satellite after the RTD. We assume that the RTD is always equal to $R T D_{\text {max }}$ in order to consider the worst case.

The main limitation for the extension of the PRMA protocol to the satellite case is that the RTD reduces the number of contention attempts within $D_{\text {max }}$. In order to overcome this problem, we consider a novel version of the PRMA protocol, named Packet Reservation Multiple Access with Hindering States (PRMA-HS), where a UT does not stop contending while it is waiting for the feedback information from the satellite. This approach assures more attempts before dropping a packet, but the drawback is that a UT may contend even if it has already obtained a reservation, because it does not yet know the outcome of its attempts. In such a case the UT is in a hindering state, because if it

\footnotetext{
* This result is consistent with that shown in Reference 21 , where $T_{\mathrm{f}}=16 \mathrm{~ms}$ was selected with slightly different system parameter values (i.e. both $p$ and the parameters of Table I).
}

attempts to transmit, it may obstruct the access of other UTs in the contending state (hindering contention). In any case, the controller onboard the satellite must check its database and discard any positive access after the first one.* Therefore the PRMA-HS protocol entails a greater number of transmission attempts than necessary. This work will prove that the advantages obtained by more frequent access attempts overcome the drawback of hindering contentions (see Section 5). This interesting result can be magnified through a suitable selection of system parameters (i.e. $p, T_{\mathrm{f}}, R T D_{\max }$ ). Note that if we apply the PRMA-HS protocol to the terrestrial case, it becomes identical to the classical PRMA protocol, since the RTD can be neglected and no hindering contention is experienced.

In investigating the performance of the classical PRMA protocol, we have assumed $T_{\mathrm{f}}=R T D_{\max }$. New we remove such a constraint by allowing $R T D_{\max } \leq T_{\mathrm{f}}$. In particular, we consider $T_{\mathrm{f}}=$ $n R T D_{\max }$, where $n$ must be greater than or equal to unity. Moreover, for the sake of simplicity we consider only integer values for $n$ which are a divisor of $N$. We expect that for a fixed value of $T_{\mathrm{f}}$ the performance (e.g. in terms of $P_{\text {drop }}$ ) can improve if the RTD is reduced.

In this study we select again $T_{\mathrm{f}}=15 \mathrm{~ms}$, the optimum value obtained for the PRMA protocol. We have verified by simulations that this is a good choice also for PRMA-HS (in Section 5 we will show that PRMA-HS in LEO-MSSs attains the same optimum capacity of PRMA in terrestrial systems if $T_{\mathrm{f}}=15 \mathrm{~ms}$ ). Therefore, according to both the constraint $T_{\mathrm{f}}=n R T D_{\max }$ and the typical values of $R T D_{\max }$ in LEO-MSSs, we have that reasonable values for $n$ are 1,2 and 3; values of $n$ greater than 3 entail an $R T D_{\max }$ value which is lower than that allowed by LEO systems. In Section 5 we evaluate the performance of both PRMA and PRMA-HS for LEO systems in the two extreme cases with $n=3$ (LEO satellites at very low altitude) and $n=1$ (LEO satellites at higher altitude); in particular, $n=3$ entails $R T D_{\max }=5 \mathrm{~ms}$ and a satellite altitude of $500 \mathrm{~km}$ for $E l_{\min }=40^{\circ}$, whereas $n=1$ entails a satellite altitude of $1600 \mathrm{~km}$ for $E l_{\min }=40^{\circ}$ (see Figure 1).

Let us describe more deeply the PRMA-HS protocol. The behaviour of each UT is modelled by a state diagram, where transitions may occur at the end of each time slot. The difference between the terrestrial PRMA and our version resides in the contending state, which must take into account both

\footnotetext{
* In this work we only envisage a voice service; then a UT only needs to obtain the reservation of a single slot per frame. On the other hand, in multimedia systems we may consider multirate applications; then also a multislot reservation depending on the type of service may be required. The investigation of such a case is beyond the scope of this paper. In any case, it is expected that PRMA-HS performs better than the original PRMA also with multirate applications, since it is possible to manage a multislot reservation for a UT.
} 
the presence of a non-negligible round-trip propagation delay (here assumed equal to $R T D_{\max }=N / n$ slots) and the behaviour of those UTs which have obtained a reservation but have not yet been informed by the controller.

A UT remains in the contending state $(\mathrm{CON})$ until it obtains a reservation; if the transmission attempt of the UT fails, it will have to contend again in the next free slot on which it has permission to transmit. We do not consider any packet capture effect (conservative assumption). ${ }^{21}$ Let us assume that only one UT has attempted transmitting in a given slot, so it will obtain the reservation of that slot, but it will know the result after an $R T D_{\max }$ time, i.e. $N / n$ slots. Before this time the UT does not know anything about its positive attempt and therefore continues to make transmission attempts on idle slots on which it has permission. This situation can be represented by a chain of $N / n$ hindering states $\left(\mathrm{HIN}_{i}\right)$.

Therefore, in order to model the PRMA-HS protocol, we consider the scheme proposed for the terrestrial PRMA in Figure $2^{21}$ and replace the CON state with a global contending state (GCON) and a special block of reservation states $\left(\mathrm{RES}_{i}^{\prime}\right)$; the resulting model has been shown in Figure 5. The GCON state is formed by those states from which a terminal may attempt a transmission, i.e. both the contending state $(\mathrm{CON})$ and the hindering states $\left(\mathrm{HIN}_{i}\right)$. The scheme in Figure 5 is related to a general value of $n$; the overall time spent in the hindering states plus the special block of reservation states $\left(\operatorname{RES}_{i}^{\prime}\right)$ must be equal to the frame duration (i.e. $N$ slots). In Figure 5 we have used some symbols that have been already introduced for the classical model of the terrestrial PRMA protocol. The justification of the GCON structure is given below.

An active UT stays in the silent state (SIL) until it has no speech packet to transmit. As soon as the first packet of a talkspurt is generated, the UT enters the CON state. The UT remains in the CON state until its attempt is successful, unless the talkspurt does not end before. With a successful attempt the UT leaves the CON state and enters the hindering states chain from $\mathrm{HIN}_{N-1}$ to $\mathrm{HIN}_{N-N / n}$; these states model the delay related to the result of the transmission attempt, which is equal to $R T D_{\max }$, i.e. $N / n$ slots. When the UT is in an $\mathrm{HIN}_{i}$ state, it may continue to attempt transmissions on available slots even if it has just obtained a reservation, because it will be notified of the success of its attempt only at the end of the $\mathrm{HIN}_{i}$ states chain. From the UT point of view the CON and the $\mathrm{HIN}_{i}$ states are indistinguishable. Then the distinction shown in Figure 5 is only possible by the controller onboard the satellite. When the UT leaves the hindering states, it receives a positive acknowledgment and must wait for $2 N / n$ slots either to transmit the next packet on the next frame or to make a transition towards the SIL state. The time of $2 N / n$ slots is spent by the UT in a special block of reservation states $\left(\mathrm{RES}_{i}^{\prime}\right)$ to wait for the right synchronism to transmit a packet on the next reserved slot.

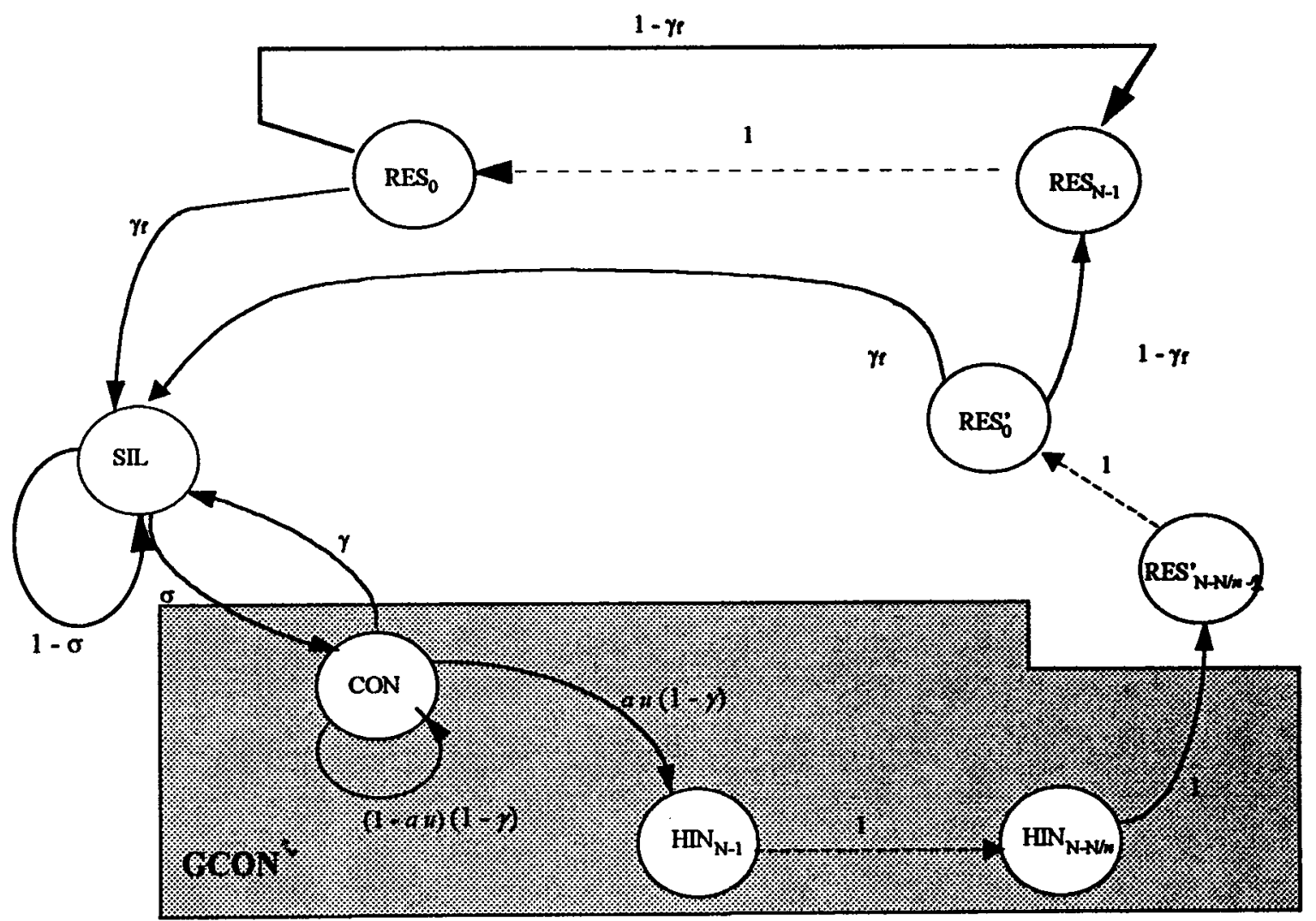

Figure 5. Terminal state diagram for PRMA-HS protocol in a satellite system with a general $n$ value 


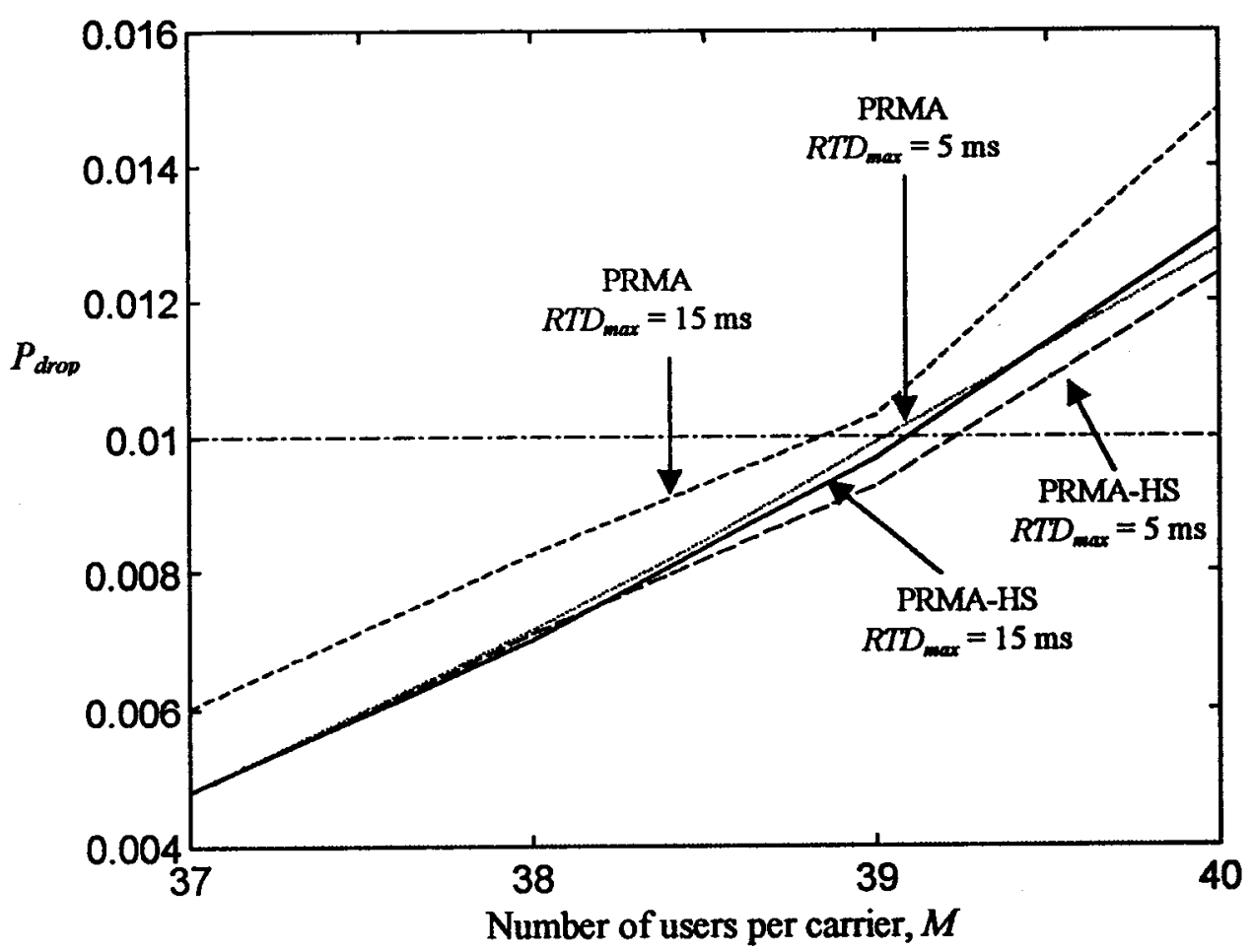

Figure 6. Comparison in terms of $P_{\text {drop }}$ between PRMA-HS protocol and original PRMA protocol in LEO-MSSs with $p=0 \cdot 4$ and $T_{\mathrm{f}}=15 \mathrm{~ms}$ for both $n=3$ (i.e. $R T D_{\max }=5 \mathrm{~ms}$ ) and $n=1$ (i.e. $R T D_{\max }=15 \mathrm{~ms}$ )

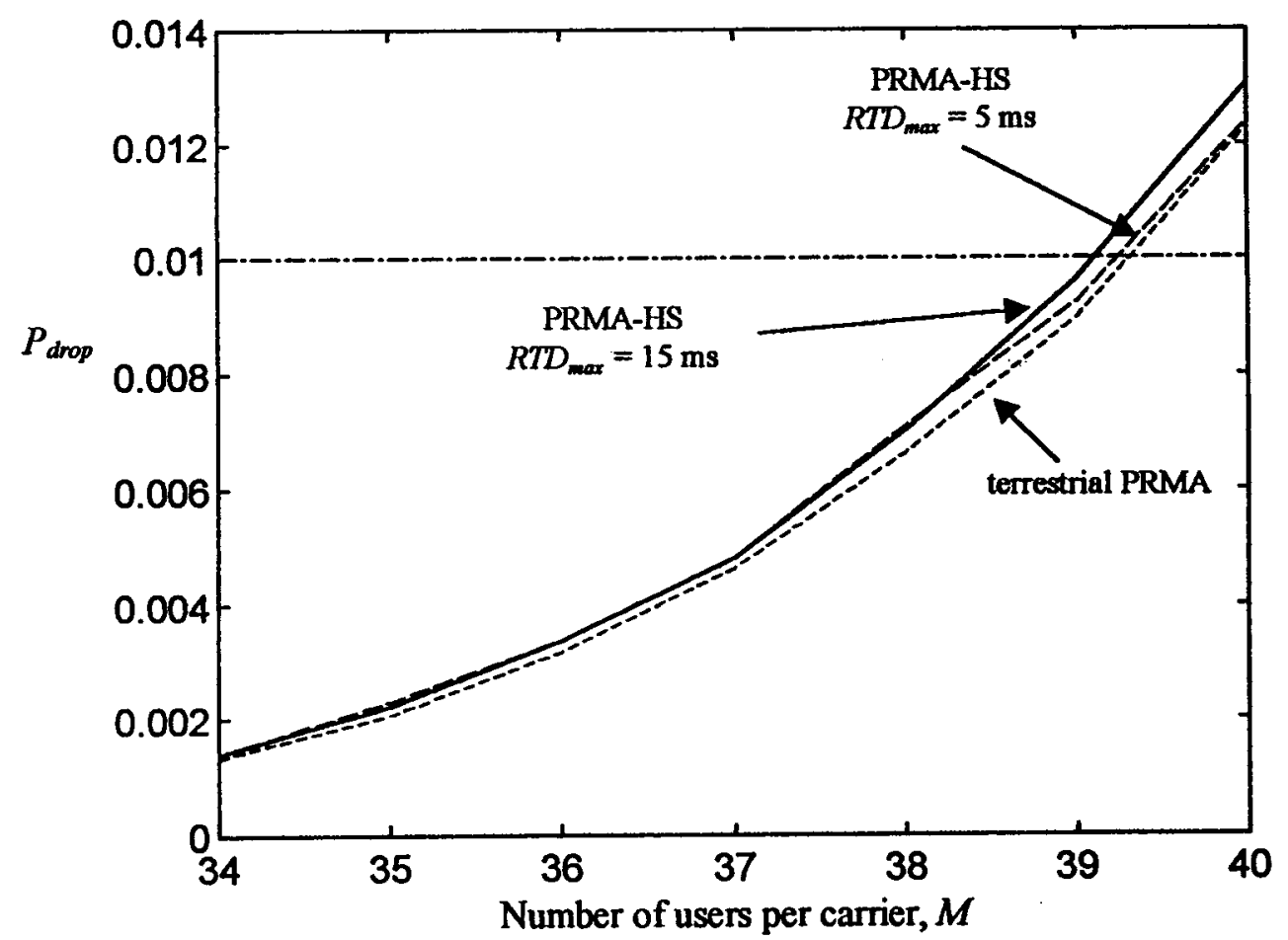

Figure 7. Behaviour of $P_{\text {drop }}$ for PRMA-HS protocol in LEO satellite case ( $R T D_{\max }=5$ and $\left.15 \mathrm{~ms}\right)$ and original PRMA protocol in terrestrial case $(\mathrm{RTD}=0)$ for $p=0.4$ and $T_{\mathrm{f}}=15 \mathrm{~ms}$

When the UT leaves the RES ${ }_{i}^{\prime}$ states, if it still has voice packets to transmit, it enters the $\operatorname{RES}_{N-1}$ to $\mathrm{RES}_{0}$ loop. When the UT is in the RES $\mathrm{R}_{0}$ state, it transmits its packet, and if it has another packet to transmit (with probability $1-\gamma_{\mathrm{f}}$, the talkspurt is not ended in $T_{\mathrm{f}}$ ), it goes back to the $\mathrm{RES}_{N-1}$ state, otherwise it leaves the RES loop and reaches the SIL state.

\section{SIMULATION RESULTS}

The aim of this section is to estimate the performance of the PRMA-HS protocol in terms of both 


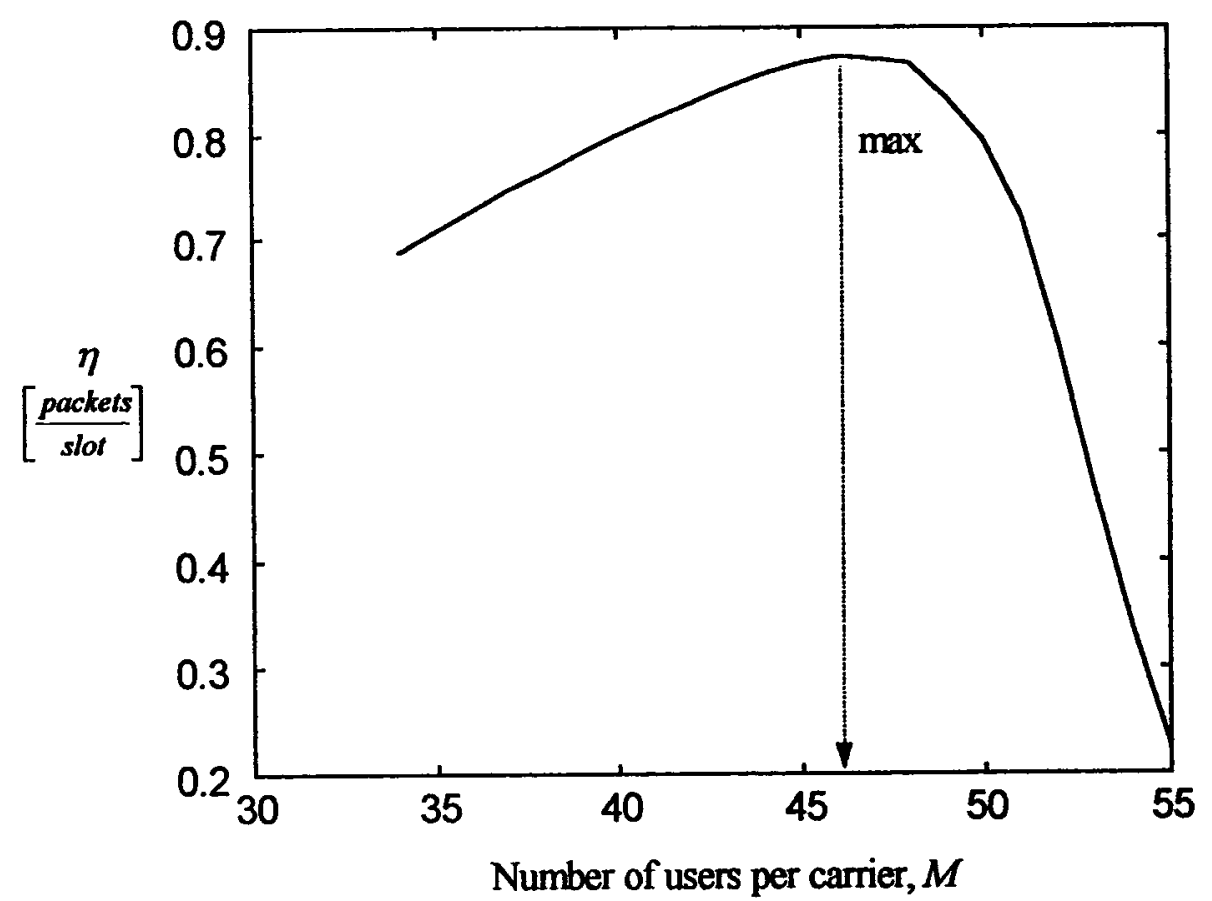

Figure 8. System throughput for PRMA-HS as a function of number of terminals, $M$, for $T_{\mathrm{f}}=15 \mathrm{~ms}, R T D_{\max }=5 \mathrm{~ms}$ and $p=0 \cdot 4$

the packet dropping probability $P_{\text {drop }}$ and the throughput $\eta$. We have assumed the system parameter values presented in Table I.

The peculiarity of PRMA-HS is that a UT does not stop contending while it waits for the result of a transmission attempt. This strategy allows the UT more transmission attempts within $D_{\max }$. The problem is that when the UT obtains a reservation, it knows this result after an $R T D_{\max }$ time. Meanwhile, other transmissions may be attempted by the UT, but they are useless. These attempts may hinder the reservation process of UTs in the CON state. Let us introduce the probability $P_{\text {hin }}$ that a transmission attempt hinders other useful transmission attempts. According to the system parameter values shown in Table I, $T_{\mathrm{f}}=15 \mathrm{~ms}$ and $n=3$, we have evaluated $P_{\text {hin }}$ by simulations for $M=40$ users/carrier. This is a quite congested situation, since $N=21$; then the impact of hindering contentions is evaluated in the worst case. We have obtained that $P_{\text {hin }} \approx 2 \%$ : among 100 attempts originated by UTs in $\mathrm{HIN}_{i}$ states, on average, only two attempts actually hinder the reservation process of other UTs in the CON state. Therefore we have proved that the drawback of hindering contentions is negligible.

Figure 6 shows the comparison between the PRMA-HS protocol and the PRMA scheme in terms of $P_{\text {drop }}$ in LEO-MSSs for both $n=3$ (i.e. $R T D_{\max }=5 \mathrm{~ms}$ ) and $n=1$ (i.e. $R T D_{\max }=15 \mathrm{~ms}$ ); moreover, we have considered homogeneous conditions for both protocols (i.e. $p=0 \cdot 4, T_{\mathrm{f}}=15 \mathrm{~ms}$ ). We have that the performance difference between PRMA and PRMA-HS is slight for $n=3$ and significant for $n=1$. Hence we may state that the performance of PRMA depends strongly on $n$ : an increase in the RTD leads to a worse behaviour; whereas PRMA-HS is less sensitive to variations of RTD. This is an interesting result that makes this protocol quite insensitive to the variations of RTD experienced in LEO systems during call lifetime, mainly due to the motion of LEO satellites. Therefore the PRMA-HS performance obtained for $n=1$ (i.e. $T_{\mathrm{f}}=R T D_{\max }$ ) can be considered as a good (conservative) estimate of the PRMA-HS performance for any $R T D_{\max }$ value less than $T_{\mathrm{f}}$.

Figure 7 presents the $P_{\text {drop }}$ behaviour of PRMAHS as a function of $M$ for both a terrestrial cellular system (where $\mathrm{RTD} \approx 0$ and PRMA-HS is identical to PRMA) and LEO-MSSs assuming $T_{\mathrm{f}}=15 \mathrm{~ms}$ and $p=0.4$; in particular, we have considered a LEOMSS with $R T D_{\max }=5 \mathrm{~ms}$ and another LEO-MSS with $R T D_{\max }=15 \mathrm{~ms}$. From this graph we may note that the PRMA-HS protocol in the LEO case with $T_{\mathrm{f}}=15 \mathrm{~ms}$ permits us to achieve the same capacity as the PRMA protocol in the terrestrial environment, i.e. 39 users/carrier (the multiplexing gain $\mu_{0.01}$ is equal to 1.63 conversations/channel).

Finally, Figure 8 shows the system throughput for PRMA-HS as a function of the number of terminals, $M$, for $T_{\mathrm{f}}=15 \mathrm{~ms}$ and $p=0.4$. We may note that the throughput has a maximum for $M \approx 45$ users/carrier. For values of $M$ greater than 45 users/carrier the throughput decreases and this behaviour highlights a system congestion. For $M=M_{0.01}$ we obtain a throughput $\eta \approx 0.75$ packets/slot; this is the same optimum performance obtained for the throughput in the terrestrial PRMA.

\section{CONCLUSIONS}

In this preliminary work we have carried out a feasibility study for the application of the PRMA 
protocol to Mobile Satellite Systems where the Round Trip Delay is not negligible with respect to the slot duration. We have shown that PRMA maintains a satisfactory performance in Low Earth Orbit systems. Then a parameter selection has been carried out for both the frame duration and the permission probability.

The subsequent step has been the proposal of an improved version of the PRMA protocol, named PRMA with Hindering States (PRMA-HS), which attains a very good performance in LEO systems and behaves like the classical PRMA scheme when $\mathrm{RTD}=0$ (i.e. in terrestrial systems). The performance of the proposed PRMA-HS protocol has been evaluated by simulations. We have obtained that the PRMA-HS scheme in LEO systems attains the same capacity and throughput as the terrestrial PRMA protocol. Thus we may conclude that the PRMAHS protocol is a good candidate as a MAC protocol in future mobile communication systems which aim to integrate a terrestrial and a satellite segment.

Further work is needed to consider integrated voice and data traffic and multirate applications. We expect that in the presence of voice and data traffic the PRMA-HS protocol will not lose its multiplexing characteristics. Moreover, other efforts should be made to take into account both the capture effect, which may reduce the packet dropping probability, and propagation impairments in LEO channels, which may obstruct the reservation mechanism. Finally, the impact of different voice codecs on the performance of the PRMA-HS protocol should be considered.

\section{acknowledgements}

This work was supported by the Italian Space Agency and MURST.

\section{REFERENCES}

1. European Commission, 'Green paper on a common approach in the field of mobile and personal communications in the European Union', 1994.

2. J. Schwarz da Silva, B. Barani and B. Arroyo-Fernandez, 'European mobile communications on the move', IEEE Commun. Mag., 34, (2), 60-69 (1996).

3. E. Del Re, 'A coordinated European effort for the definition of a satellite integrated environment for future mobile communications', IEEE Commun. Mag., 34, (2), 98-104 (1996).
4. G. Maral, J. J. De Ridder, B. G. Evans and M. Richharia, 'Low earth orbit satellite systems for communications', Int. J. Satell. Commun., 9, 209-225 (1991).

5. D. J. Goodman, R. A. Valenzuela, K. T. Gayliard and B. Ramanurthi, 'Packet Reservation Multiple Access for local wireless communications', IEEE Trans. Commun., COM-37, 885-890 (1989).

6. D. J. Goodman, 'Trends in cellular and cordless communications', IEEE Commun. Mag., 29, (6), 31-40 (1991).

7. S. Nanda, 'Stability evaluation and design of the PRMA joint voice data system', IEEE Trans. Commun., COM-42, 2092-2104 (1994)

8. W. C. Wong and D. J. Goodman, 'Integrated data and speech transmission using Packet Reservation Multiple Access', Proc. ICC'94, 1994, pp. 172-176.

9. SAINT Project (RACE II), Deliverable 11, 'Radio resource management', R2117-FIU-DIE.DR.P211-B1, 1995.

10. M. Frullone, G. Falciasecca, P. Grazioso, G. Riva and A. M. Serra, 'On the performance of Packet Reservation Multiple Access with fixed and dynamic channel allocation', IEEE Trans. Vehic. Technol., VT-42, 78-86 (1993).

11. W.-C. Wong, 'Dynamic allocation of Packet Reservation Multiple Access carriers', IEEE Trans. Vehic. Technol., VT42, 385-392 (1993).

12. Y. Li, S. Andersen and B. Feng, 'On the performance analysis of EPRMA protocol with Markov chain model', Proc. GLOBECOM'95, Singapore, November 1995, pp. $1502-1506$.

13. A Urie, 'ATDMA project and system', Proc. 2nd Joint COST 227/231 Workshop on Mobile and Personal Communications, Florence, April 1995, pp. 11-22.

14. X.-F. Dong and L.-M. Li, 'A spread spectrum PRMA protocol with randomized arrival times for microcellular systems', Proc. GLOBECOM'96, London, November 1996.

15. G. Bianchi, F. Borgonovo, L. Fratta, L. Musumeci and M. Zorzi, 'C-PRMA: a centralized Packet Reservation Multiple Access for local wireless communications', IEEE Trans. Vehic. Technol., VT-46, 422-436 (1997).

16. V. H. Bharghava, D. Haccount, R. Matyas and P. P. Nuspl, Digital Communications by Satellite, Wiley, New York, 1981.

17. Web page with address: http://www.teledesic.com.

18. Web page with address: http://www.iridium.com.

19. E. Del Re, R. Fantacci and G. Giambene, 'Efficient dynamic channel allocation techniques with handover queuing for mobile satellite networks', IEEE J. Select. Areas Commun., SAC-13, 397-405 (1995).

20. L. G. Roberts, 'ALOHA packet system with and without slots and capture', Comput. Commun. Rev., 5, 28-42 (1975).

21. S. Nanda, D. J. Goodman and U. Timor, 'Performance of PRMA: a packet voice protocol for cellular systems', IEEE Trans. Vehic. Technol., VT-40, 584-598 (1991).

22. J. Gruber and L. Strawczynski, 'Subjective effects of variable delay and speech clipping in dynamically managed voice systems', IEEE Trans. Commun., COM-38, 801-808 (1990).

23. A. Fukuda and S. Tasaka, 'The equilibrium point analysisa unified analytic tool for packet broadcast networks', Proc. GLOBECOM'83, San Diego, November 1983, pp. 33.4.133.4.8.

24. S. Tasaka, 'Performance comparison of multiple access protocols for satellite broadcast channels', Proc. GLOBECOM'83, San Diego, CA, November 1983, pp. 35.3.1-35.3.8.

25. F. Ananasso and F. Delli Priscoli, 'The role of satellites in personal communication services', IEEE J. Select. Areas Commun., SAC-13, 180-196 (1995). 